\title{
FDA-Approved Drugs Efavirenz, Tipranavir, and Dasabuvir Inhibit Replication of Multiple Flaviviruses in Vero Cells
}

\author{
Michal Stefanik ${ }^{1,2}$, James J. Valdes ${ }^{1,3}$, Fortunatus C. Ezebuo ${ }^{4}{ }^{\oplus}$, Jan Haviernik ${ }^{1,5}$, \\ Ikemefuna C. Uzochukwu ${ }^{4} \mathbb{D}^{D}$, Martina Fojtikova ${ }^{1}$, Jiri Salat ${ }^{1}$, Ludek Eyer ${ }^{1,3}$ and \\ Daniel Ruzek 1,3,*(D) \\ 1 Department of Virology, Veterinary Research Institute, Hudcova 70, CZ-62100 Brno, Czech Republic; \\ stefanik@vri.cz (M.S.); valdjj@gmail.com (J.J.V.); haviernik@vri.cz (J.H.); fojtikovamartina@seznam.cz (M.F.); \\ salat@vri.cz (J.S.); eyer@vri.cz (L.E.) \\ 2 Department of Chemistry and Biochemistry, Mendel University in Brno, Zemedelska 1, \\ CZ-613 00 Brno, Czech Republic \\ 3 Institute of Parasitology, Biology Centre of the Czech Academy of Sciences, Branisovska 31, \\ CZ-37005 Ceske Budejovice, Czech Republic \\ 4 Department of Pharmaceutical and Medicinal Chemistry, Faculty of Pharmaceutical Sciences, Nnamdi \\ Azikiwe University, PMB 5025 Awka 420281, Nigeria; fortunatus.ezebuo@unn.edu.ng (F.C.E.); \\ ic.uzochukwu@unizik.edu.ng (I.C.U.) \\ 5 Faculty of Science, Masaryk University, Kamenice 5, CZ-625 00 Brno, Czech Republic \\ * Correspondence: ruzekd@paru.cas.cz
}

Received: 10 February 2020; Accepted: 15 April 2020; Published: 20 April 2020

\begin{abstract}
Vector-borne flaviviruses (VBFs) affect human health worldwide, but no approved drugs are available specifically to treat VBF-associated infections. Here, we performed in silico screening of a library of U.S. Food and Drug Administration-approved antiviral drugs for their interaction with Zika virus proteins. Twelve hit drugs were identified by the docking experiments and tested in cell-based antiviral assay systems. Efavirenz, tipranavir, and dasabuvir at micromolar concentrations were identified to inhibit all VBFs tested; i.e., two representatives of mosquito-borne flaviviruses (Zika and West Nile viruses) and one representative of flaviviruses transmitted by ticks (tick-borne encephalitis virus). The results warrant further research into these drugs, either individually or in combination, as possible pan-flavivirus inhibitors.
\end{abstract}

Keywords: FDA; flavivirus; Zika virus; tick-borne encephalitis virus; West Nile virus; antiviral

\section{Introduction}

The genus Flavivirus (family Flaviviridae) comprises more than 50 members, most of which are transmitted by mosquitoes and ticks (vector-borne flaviviruses, VBF) [1]. Despite similarities in genomic organization, replication strategy, and physicochemical properties, flaviviruses can cause a variety of diseases with clinical presentations ranging from mild fever to hemorrhagic fever, encephalitis, Guillain-Barré syndrome, and microcephaly [2]. Important human pathogens include yellow fever virus, dengue virus, West Nile virus (WNV), Zika virus (ZIKV), Japanese encephalitis virus, and tick-borne encephalitis virus (TBEV) [3,4]. No approved effective antiviral therapy directed against these viruses is currently available. To address this urgent medical need, we interrogated a library of U.S. Food and Drug Administration (FDA)-approved antiviral drugs for the ability to block flavivirus replication in vitro. Such approved drugs have well-documented modes of action, safety, and pharmacokinetic and pharmacodynamic profiles. Therefore, identifying them might expedite the regulatory process for their approval in clinical use more rapidly than new compounds [5-9]. 
In this study, we first performed in silico screening of a library of FDA-approved antiviral drugs for their interaction with ZIKV proteins (NS3 helicase and protease, NS5 RNA-dependent RNA polymerase, and methyltransferase). The cytotoxicities and antiviral activities of the identified hit compounds were tested against three representative flaviviruses: ZIKV and WNV as emerging mosquito-borne pathogens, and TBEV as an important tick-borne pathogen. Our results identified three FDA-approved drugs - efavirenz (an antiretroviral drug that targets the HIV-1 reverse transcriptase enzyme), tipranavir (a nonpeptidic protease inhibitor that targets the HIV protease), and dasabuvir (an inhibitor of NS5B polymerase, terminating RNA polymerization and stopping the replication of the genome of hepatitis $C$ virus) - that inhibit flavivirus infection in vitro. To the best of our knowledge, none of these three drugs have been previously reported to have anti-VBF activity.

\section{Materials and Methods}

\subsection{In Silico Screen of the Library of FDA-Approved Drugs}

Bioinformatics mining of the Protein Data Bank (PDB) was done to identify ZIKV proteins whose 3D structures have been deposited. The 3D atomic coordinates of six identified ZIKV protein structures (NS3 helicase (5K8T), protease (5H6V), and NS5 methyltransferase (5MRK, 5KQS, and 5ULP)) and RNA-dependent RNA polymerase (5U04) were obtained from PDB [10] and prepared for molecular docking simulation using UCSF Chimera 1.9 [11] and AutoDockTools 1.5.6 [12,13]. Briefly, all duplicate chains and hetero molecules were deleted, and polar hydrogen atoms were added. Grid box sizes, centers, and exhaustiveness were assigned to the proteins at $1.0 \AA$, as shown in Table 1 . Respective pdbqt files were created for molecular docking simulations studies.

Table 1. Grid box centers and sizes used for molecular docking simulations.

\begin{tabular}{|c|c|c|c|c|c|c|c|}
\hline \multirow[t]{2}{*}{ Protein } & \multicolumn{3}{|c|}{ Center } & \multicolumn{3}{|c|}{ Size } & \multirow[t]{2}{*}{ Exhaustiveness } \\
\hline & $\mathbf{x}$ & $\mathbf{y}$ & $\mathbf{z}$ & $\mathbf{x}$ & $\mathbf{y}$ & $\mathbf{z}$ & \\
\hline 5MRK & 18.216 & 7.699 & 4.793 & 13 & 18 & 15 & 8 \\
\hline $5 \mathrm{~K} 8 \mathrm{~T}$ & 115.969 & 2.824 & 64.433 & 14 & 19 & 14 & 8 \\
\hline $5 \mathrm{H} 6 \mathrm{~V}$ & -8.522 & 3.178 & -14.473 & 12 & 10 & 17 & 8 \\
\hline $5 \mathrm{KQS}$ & 52.145 & 10.164 & -2.722 & 15 & 15 & 12 & 8 \\
\hline 5ULP & -2.874 & -1.66 & 26.51 & 15 & 17 & 16 & 8 \\
\hline 5U04 & 25.036 & 68.817 & 103.577 & 12 & 16 & 16 & 8 \\
\hline
\end{tabular}

Key: methyltransferases (5MRK, 5KQS, and 5ULP), NS3 helicase (5K8T), protease (5H6V), NS5 RNA-dependent RNA polymerase (5U04).

A library of 1960 FDA-approved drugs were obtained from Drug Bank [14] as of July 6, 2017. From this library, 73 were identified as FDA-approved antiviral drugs. These antiviral drugs were converted to their respective 3D coordinates with Open Babel 2.3.0 [15] and prepared for molecular docking simulation using AutoDockTools 1.5.6 [12,13]. Briefly, rotatable bonds were determined, all hydrogens were added, Gasteiger charges were computed, and pdbqt files were created for docking simulations studies. The prepared receptors and drugs then were used for the molecular docking simulation.

To validate the molecular docking simulations protocol, the experimental complexes of sinefungin (SFG), 7N-methyl-8-hydroguanosine-5'-diphosphate (M7G), 5'-\{[(3s)-3-amino-3carboxypropyl][(4-fluorophenyl)methyl]amino\}-5'-deoxyadenosine (KBI), 5'-guanosinediphosphate-monothiophosphate (GSP), and (s)-2-acetamido-6-amino-n-((s)-5-guanidino-1oxopentan-2-yl) hexanamide (7HS) with their ZIKV target proteins (NS5 methyltransferase (5MRK, $5 \mathrm{KQS}, 5 \mathrm{ULP})$, NS3 helicase (5K8T), and protease (5H6V)) from PDB were reproduced in silico after ligands were obtained from the ZINC ${ }^{\circledR}$ database [16] or extracted from the protein and subjected to geometry optimization. Blind docking was first performed with $5 \mathrm{U} 04$ and subsequently validated using M7G. AutoDockVina ${ }^{\circledR}$ has a high accuracy in predicting binding free energies by setting the 
receptor rigid while appraising flexible ligands with a comparatively low standard error $[17,18]$. Therefore, receptor conformational flexibilities were neglected by rigid receptor docking. The approved drugs were docked into the receptors using AutoDockVina ${ }^{\circledR}$ after the validation of molecular docking protocols. The simulations were performed locally on a Linux platform using a configuration file and script (Supplementary Material S1) containing information on the grid box centers and sizes (Table 1) of prepared receptors and selected drugs.

\subsection{Induced Fit Simulations}

The ZIKV polymerase, PDB: 5U0B without its methyltrasferase domain (residues 1-267), and the ZIKV protease, PDB: 5YOF, were used for molecular docking, as previously indicated, with an increased box size of $25_{x y z}$. These ZIKV structures were used since they are at higher resolution with no missing residues. The docked ZIKV enzymes were then prepared and optimized for induced fit simulations using the Schrödinger's Maestro Protein Preparation Wizard [19]. Steric clashes were eliminated via local minimization. Note that the Maestro Protein Preparation Wizard created protonation states during optimization (default) that were used in the induced fit simulations.

The induced fit simulations were performed using the Metropolis Monte Carlo-based Protein Energy Landscape Exploration server (PELE). The PELE server is freely available at pele.bsc.es and has been previously explained [20,21]. Briefly, the PELE software executes three steps: (1) a protein/ligand local perturbation, (2) a residue side chain sampling, and (3) a global minimization. The three steps are repeated for a number of iterations within $24 \mathrm{~h}$. The Metropolis Monte Carlo-based method employed by PELE accepts iterations if the enthalpy of evaporation is equal to or less than its initial value. An iteration is rejected if the enthalpy is greater than its initial value. The change in enthalpy $(\Delta H)$ is calculated by the force field known as the optimized potentials for liquid simulations (OPLS-2005) [22]. The inhibitor binding enthalpy $\left(\Delta \mathrm{H}_{\mathrm{L}}\right)$ is calculated according to Equation (1):

$$
\Delta \mathrm{H}_{\mathrm{L}}=\Delta \mathrm{H}_{\mathrm{ab}}-\left(\Delta \mathrm{H}_{\mathrm{a}}+\Delta \mathrm{H}_{\mathrm{b}}\right)
$$

where $\Delta \mathrm{H}_{\mathrm{ab}}$ is the enthalpy of the entire system, including inhibitors, and $\Delta \mathrm{H}_{\mathrm{a}}$ and $\Delta \mathrm{H}_{\mathrm{b}}$ are their separate enthalpy values.

For this study, the PELE ligand refinement ready-made script was used on the re-docked apo-enzymes for initial induced fit simulations. Alterations to the ready-made script were how long the inhibitor explores a region (wait for $=5$ ) and the region explored (spawn within $=2-7 \AA$ ). The induced fit simulations were repeated for the narrow active site and binding cavities of the ZIKV polymerase until the inhibitor approximates its coordinates within $4.5 \AA$. The best pose/iteration of the ZIKV polymerase simulations that approximates the coordinates was chosen for the following two steps. (1) The best pose/iteration was refined using the PELE protein motion ready-made script. (2) Multiple polymerase dockings were conducted using top representatives in an ensemble cluster (UCSF Chimera 1.9 [11]) from the protein motion simulation. For all ZIKV enzymes, pose/iteration that approximates its respective inhibitor coordinates was chosen for several rounds of induced fit simulations until the inhibitors approached $<1 \AA$. The modifications made on the ligand refinement ready-made script for these subsequent induced fit simulations are in Supplementary Material S2. Finally, the PELE protein motion ready-made script was used to calculate the average $\Delta \mathrm{H}_{\mathrm{L}}$ and positions of analogous/identical co-crystalized inhibitors and were compared with protein motion simulations of the ZIKV enzymes from the pose/iteration $<1 \AA$. The first 20 iterations were eliminated since the simulation reached equilibrium at this point.

\subsection{Viruses, Cells, and Tested Compounds}

The following viral strains were tested: WNV (strains Eg101, a member of genomic lineage 1 isolated from human serum in Egypt; and 13-104, a representative of genomic lineage 2 isolated from the Culex modestus mosquito in the Czech Republic), TBEV (strain Hypr, highly pathogenic 
representative of the European subtype of TBEV), and ZIKV (MR-766, a representative of the African ZIKV lineage; and Paraiba_01, a member of the Asian ZIKV lineage).

Vero cells (ATCC CCL-81, African Green Monkey, adult kidney, epithelial) were cultured in Dulbecco's Modified Eagle Medium containing 10\% fetal bovine serum, 1\% L-glutamine, $100 \mathrm{U} / \mathrm{mL}$ penicillin, and $100 \mu \mathrm{g} / \mathrm{mL}$ streptomycin (Sigma-Aldrich, Prague, Czech Republic) at $37{ }^{\circ} \mathrm{C}$ in a $5 \%$ $\mathrm{CO}_{2}$ atmosphere. PS cells (porcine kidney stable) were cultured at $37^{\circ} \mathrm{C}$ in Leibovitz (L-15) medium supplemented with $3 \%$ fetal bovine serum, $100 \mathrm{U} / \mathrm{mL}$ penicillin, $100 \mu \mathrm{g} / \mathrm{mL}$ streptomycin, and $1 \%$ L-glutamine (Sigma-Aldrich, Prague, Czech Republic). Human brain cortical astrocytes (HBCAs; ScienCell, Carlsbad, CA, USA) were cultivated at $37^{\circ} \mathrm{C}$ under $5 \% \mathrm{CO}_{2}$ atmosphere in Astrocyte medium (ScienCell, Carlsbad, CA, USA), supplemented with $6 \%$ fetal bovine serum, $100 \mathrm{U} / \mathrm{mL}$ penicillin, $100 \mu \mathrm{g} / \mathrm{mL}$ streptomycin (Sigma-Aldrich), and 1\% astrocyte growth supplement (ScienCell, Carlsbad, CA, USA). Human neuroblastoma UKF-NB-4 cells were cultured at $37{ }^{\circ} \mathrm{C}$ and $5 \% \mathrm{CO}_{2}$ atmosphere in Iscove's Modified Dulbecco's Medium, supplemented with $10 \%$ fetal bovine serum, $100 \mathrm{U} / \mathrm{mL}$ penicillin, $100 \mu \mathrm{g} / \mathrm{mL}$ streptomycin, and 1\% L-glutamine (Sigma-Aldrich, Prague, Czech Republic).

Paritaprevir, dolutegravir, raltegravir potassium, elvitegravir, efavirenz, and tauroursodeoxycholate sodium were obtained from Sigma-Aldrich (St. Louis, MO, USA) and delavirdine mesylate, tipranavir, dasabuvir (ABT-333), saquinavir mesylate, maraviroc, and trifluridine were obtained from ChemScene, LLC (Monmouth Junction, NJ, USA). 7-deaza-2'-C-methyladenosine was purchased from Carbosynth (Compton, United Kingdom). Compounds were solubilized in dimethyl sulfoxide (DMSO; 100\% v/v) to make stock solution with a concentration of $10 \mathrm{mM}$.

\subsection{Cytotoxicity Assay}

For detailed cytotoxicity studies, Vero, HBCA, and UKF-NB-4 cells were seeded onto 96-well plates at a density of 10,000 cells per well and incubated for $24 \mathrm{~h}$ at $37^{\circ} \mathrm{C}$ before being used in the experiment to form a confluent monolayer. After the incubation, tested compounds were added to the cells at concentrations of $0,3.125,6.25,12.5,25,50,75$, and $100 \mu \mathrm{M}$, and treated under the same regime as during antiviral testing; i.e., pretreatment for $24 \mathrm{~h}$ with culture medium containing appropriate drug concentrations, then the medium was exchanged with fresh compound-containing medium. After $48 \mathrm{~h}$ post medium exchange, cytotoxicity was measured using the Cell Counting Kit-8 (Dojindo Molecular Technologies, Inc., Munich, Germany) according to the manufacturer's instructions. The $50 \%$ cytotoxic concentration $\left(\mathrm{CC}_{50}\right)$ values, representing the concentration of compound that reduced cell viability by $50 \%$, were calculated using GraphPad Prism (version 7.04, GraphPad Software, San Diego, CA, USA) as a nonlinear regression (inhibitor vs. normalized response, variable slope). All assays were performed in three independent experiments done in triplicate.

\subsection{Antiviral Assays}

\subsubsection{Inhibition of ZIKV-Mediated Cytopathic Effect in a Simultaneous Treatment Assay}

Twelve compounds that were found to bind with a high affinity to the selected ZIKV proteins in the in silico simulation experiment (paritaprevir, dolutegravir, raltegravir, efavirenz, elvitegravir, tipranavir, saquinavir, dasabuvir, delavirdine, maraviroc, trifluridine, and sodium tauroursodeoxycholate) were first screened for their ability to inhibit the cytopathic effect (CPE) mediated by ZIKV (strain MR-766) infection in Vero cells. In this initial screening, all compounds were tested at a single concentration of $50 \mu \mathrm{M}$. DMSO was added to virus-infected cells as a negative control at a concentration corresponding to a dilution of the initial drug-DMSO stock (at a maximal final concentration of $0.5 \%$ $(v / v)$ ). Culture medium containing appropriate drug concentrations and simultaneously inoculated with virus (multiplicity of infection $=0.1$ ) was added to the cell monolayers. After $48 \mathrm{~h}$ of incubation at $37^{\circ} \mathrm{C}$, culture media were collected, and CPE was quantified using the Cell Counting Kit-8 (Dojindo Molecular Technologies, Inc., Munich, Germany) and expressed as percentage of cell viability. All assays were performed in three independent experiments done in triplicate. 


\subsubsection{Anti-ZIKV Activity in a Simultaneous Treatment Assay}

Compounds that reduced ZIKV-mediated CPE (>90\% viability of the cells in the culture compared to the uninfected cells) were further investigated for their activity to inhibit ZIKV growth in Vero cells. The compounds were tested at a single concentration of $50 \mu \mathrm{M}$. DMSO was added to virus-infected cells as a negative control at a concentration corresponding to a dilution of the initial drug-DMSO stock (at a maximal final concentration of $0.5 \%(v / v)$ ). Culture medium containing appropriate drug concentrations and simultaneously inoculated with virus (strain MR-766, multiplicity of infection = 0.1 ) was added to the cell monolayers. After $48 \mathrm{~h}$ of incubation at $37^{\circ} \mathrm{C}$, culture media were collected, and subjected to plaque assay as described above. All assays were performed in three independent experiments done in triplicate.

\subsubsection{Anti-ZIKV Activity in a Post-Treatment Assay}

Dasabuvir, efavirenz, and tipranavir (i.e., drugs that inhibited ZIKV in a simultaneous treatment assay) were further used in a post-treatment antiviral study. The potency of these compounds to inhibit ZIKV $2 \mathrm{~h}$ post-infection was assayed in Vero cells. The cells were infected with ZIKV (strain MR-766) at multiplicity of infection $=0.1$. After $2 \mathrm{~h}$, the medium containing virus was removed and replaced with a fresh medium containing the tested compounds at concentration of $50 \mu \mathrm{M}$. DMSO was added to virus-infected cells as a negative control at a concentration corresponding to a dilution of the initial drug-DMSO stock (at a maximal final concentration of $0.5 \%(v / v)$ ). After $48 \mathrm{~h}$ of incubation, culture media were harvested and subjected to plaque assay. 7 -deaza- $2^{\prime}-C$-methyladenosine was used at the same concentration and treatment regime as a reference compound. All assays were performed in three independent experiments done in triplicate.

\subsubsection{Anti-VBFs Activity in a Pretreatment Assay, Dose Response Study}

Dose response studies of dasabuvir, efavirenz, and tipranavir were done in Vero cells infected with TBEV, WNV, and ZIKV. The antiviral effects of dasabuvir and efavirenz were evaluated at compound concentrations of $0,12.5,25,30,40$, and $50 \mu \mathrm{M}$; tipranavir was tested at $0,25,40,50,75$, and $100 \mu \mathrm{M}$. The cells were pretreated with tested compounds for $24 \mathrm{~h}$. Then the medium was removed, and the cells were infected with the individual viruses at a multiplicity of infection of 0.1 in culture media containing appropriate drug concentration. The compound inhibitory effect was assayed against WNV (strains Eg101 and 13-104), TBEV (strain Hypr), and ZIKV (strains MR-766 and Paraiba_01). DMSO was added to virus-infected cells as a negative control at a concentration corresponding to a dilution of the initial drug-DMSO stock (at a maximal final concentration of $0.5 \%(v / v)$ ). After $48 \mathrm{~h}$ of incubation at $37^{\circ} \mathrm{C}$, culture media were collected, and viral titer was quantified by plaque assay. All assays were performed in three independent experiments done in triplicate.

\subsubsection{Anti-ZIKV Activity in Human Neural Cells a Pretreatment Assay}

For further characterization of the compound-mediated anti-ZIKV effect, we used UKF-NB-4 and HBCA cells, as target cells of neural origin. The cells were pretreated with serial dilutions of the drugs for $24 \mathrm{~h}$. The highest drug concentration represented the highest non-toxic ( $>90 \%$ viability of the treated cells) concentrations for the particular cell type (for HBCA: $12.5 \mu \mathrm{M}$ dasabuvir, $12.5 \mu \mathrm{M}$ tipranavir, $3.125 \mu \mathrm{M}$ efavirenz; for UKF-NB-4: $6.25 \mu \mathrm{M}$ dasabuvir, $50 \mu \mathrm{M}$ tipranavir, $12.5 \mu \mathrm{M}$ efavirenz). Then the medium was removed, and the cells were infected with Paraiba_01 strain of ZIKV at a multiplicity of infection of 0.1 in culture media containing appropriate drug concentration. DMSO was added to virus-infected cells as a negative control at a concentration corresponding to a dilution of the initial drug-DMSO stock (at a maximal final concentration of $0.5 \%(v / v)$ ). After $48 \mathrm{~h}$ of incubation at $37^{\circ} \mathrm{C}$, culture media were collected, and viral titer was quantified by plaque assay. All assays were performed in three independent experiments done in triplicate. 


\subsection{Plaque Assay}

Plaque assays were performed in Vero cells (for ZIKV and WNV titers) or in the PS cells (to determine TBEV titers) as described previously [23-25]. The obtained viral titer values were recalculated to percentages of viral titer inhibition, applied to constructing the dose-response and inhibition curves, and used to calculate the $50 \%$ effective concentration $\left(\mathrm{EC}_{50}\right)$. We calculated $\mathrm{EC}_{50}$ values using $\mathrm{GraphPad}$ Prism as a nonlinear regression (agonist vs. normalized response) from three independent experiments done in triplicate.

\subsection{Immunofluorescence Staining of Viral Antigen}

The results obtained from antiviral assays were confirmed using a cell-based flavivirus immunostaining assay with a mouse monoclonal antibody that specifically recognizes the flavivirus group surface antigen, as described previously [25]. Briefly, Vero cells seeded onto 96-well plates were treated with the test compound $(0,40$, or $50 \mu \mathrm{M}$ dasabuvir or efavirenz; 0,75 , or $100 \mu \mathrm{M}$ tipranavir $)$ and infected with the individual viruses at a multiplicity of infection of 0.1 . After incubation at $37^{\circ} \mathrm{C}$ for $48 \mathrm{~h}$, the cell monolayers were fixed with cold acetone-methanol (1:1), blocked with 10\% fetal bovine serum, and incubated with the flavivirus antibody (1:250; Sigma-Aldrich, Prague, Czech Republic). After washing, the cells were labeled with an anti-mouse goat secondary antibody conjugated with fluorescein isothiocyanate (FITC; 1:500) and counterstained with DAPI (4' ,6-diamidino-2-phenylindole; $1 \mu \mathrm{g} / \mathrm{mL}$ ) to allow visualization of the cell nuclei. The fluorescence signal was recorded with an Olympus IX71 epifluorescence microscope and processed by ImageJ software.

\section{Results and Discussion}

To date, there are no specific antivirals available for clinical use with activity against VBFs—excluding preclinical studies of small molecules with known anti-ZIKV properties [7]. To address this gap, and to identify large molecule drugs, we conducted an in silico screen of an FDA-approved library for antiviral drugs using ZIKV protein structures as a VBF-representative model (Table 1). These are the initial steps in our computational and biological workflow (Figure 1). From the in silico screening, we identified 12 of the 73 antiviral drugs with favorable docking scores. The 12 FDA-approved antiviral drugs are paritaprevir, dolutegravir, raltegravir, efavirenz, elvitegravir, tipranavir, saquinavir, dasabuvir, delavirdine, maraviroc, trifluridine, and sodium tauroursodeoxycholate. These 12 in silico selected antiviral drugs were then tested in vitro for an anti-ZIKV effect on Vero cells at a concentration of $50 \mu \mathrm{M}$ (simultaneous treatment assay). Inhibition of ZIKV-induced CPE was monitored by light microscopy and quantified at $48 \mathrm{~h}$ after infection using the in vitro assay for quantitative evaluation of the cell viability, as previously described [26]. From the in vitro screening, four antivirals (efavirenz, tipranavir, dolutegravir, and dasabuvir) inhibited ZIKV-mediated CPE in cell culture ( $>90 \%$ viability of the infected cells compared to uninfected controls) at a concentration of $50 \mu \mathrm{M}$ (Figure 2A). However, only three of these (efavirenz, tipranavir, and dasabuvir) reduced ZIKV titer in the culture at this concentration (Figure 2B). The antiviral effect of efavirenz, tipranavir, and dasabuvir was further demonstrated in another experiment, when these drugs were applied to ZIKV-infected Vero cells $2 \mathrm{~h}$ post-infection (post-treatment assay; Supplementary Figure S1). The anti-ZIKV effect of tipranavir and dasabuvir was even stronger compared to 7-deaza-2'-C-methyladenosine, which is known to be an effective ZIKV inhibitor with documented activity both in vitro [26] as well as in vivo [27], and was used as a reference compound in our study (Supplementary Figure S2). 


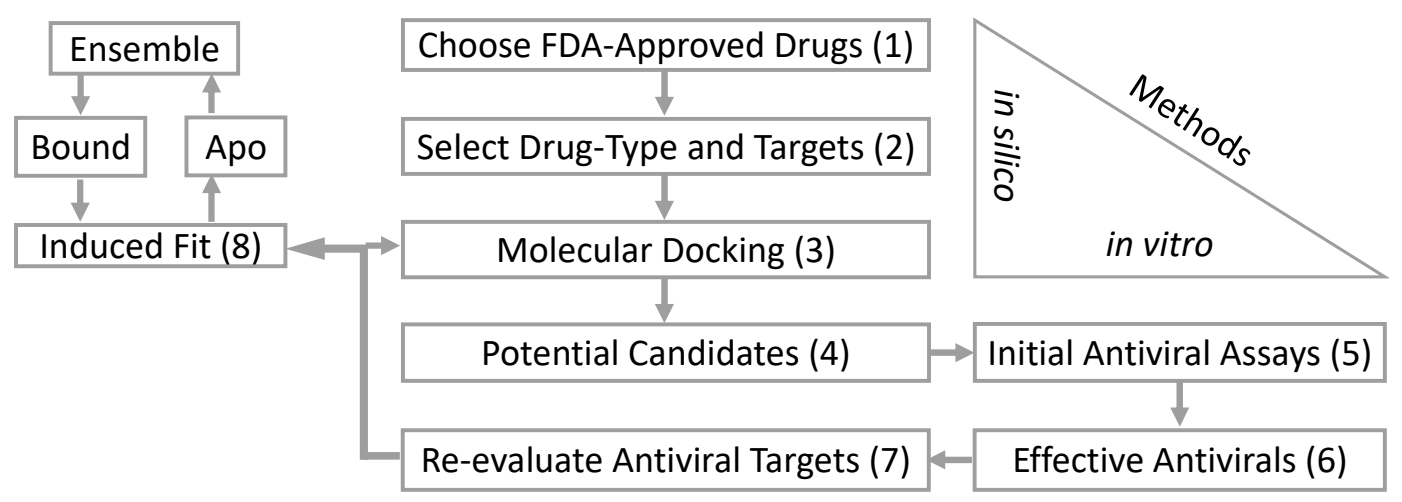

Figure 1. The workflow schematic indicates that a total of 1960 U.S. Food and Drug Administration (FDA)-approved drugs were downloaded from Drug Bank in the year 2017 (1). Excluding FDA-approved small molecule antiviral drugs ( $<900$ daltons), only 73 were $>1000$ daltons. These 73 antivirals were then screened in silico with six Zika virus (ZIKV) proteins (2; Table 1). After the molecular docking screen (3), 12 FDA-approved antiviral drugs resulted in favorable scores (4). An in vitro assay of these 12 antivirals (5) resulted in three effective anti-ZIKV FDA-approved drugs (6). The drugs and targets were re-evaluated by searching the Protein Data Bank (PDB) for similar co-crystalized compounds (7). The molecular dockings were repeated using the average coordinates from various inhibitors. Finally, a series of induced fit simulations and dockings (8) were conducted on the favorable re-docked structures. The structures were then compared to similar co-crystalized compounds.

According to the initial molecular docking results, dasabuvir and tipranavir bind to the ZIKV methyltransferase (PDB: 5MRK) and efavirenz binds to ZIKV protease (PDB: 5H6V). These are apparent false positives since the non-nucleoside efavirenz and the non-peptidomimetic tipranavir have co-crystalized structures with HIV polymerase [28] and HIV protease [29], respectively. To date, there are no co-crystalized structures with dasabuvir. As a non-nucleoside inhibitor, however, dasabuvir is known to interact with hepatitis C (HCV) polymerase [30]. Given the dearth of ZIKV structures co-crystalized with non-nucleoside and non-peptidomimetic inhibitors, and the ZIKV structural diversity with HIV enzymes, the three effective ZIKV FDA-approved antivirals were therefore re-evaluated for subsequent molecular docking.

A PDB search resulted in $16 \mathrm{HCV}$ and three Dengue polymerases co-crystalized with non-nucleoside inhibitors. Among the HCV polymerases, the non-nucleoside inhibitor 28V (PubChem ID: 46220530) is similar in composition to dasabuvir. A similar PDB search did not reveal any flavivirus proteases co-crystalized with non-peptidomimetic inhibitors. We therefore used the positions of two macrocyclic HCV protease inhibitors. The PDB accession numbers are in Supplementary Table S1. The average positions of the binding coordinates are shown with the ZIKV structures (Figure 3A,B). The ZIKV polymerase and protease, with respective inhibitors, were prepared for molecular docking as previously indicated. Conformational changes between bound and apo-enzymes at the binding site are necessary for an accurate docking pose. The top docking poses that approximate their respective average binding coordinates (Figure $3 \mathrm{~A}, \mathrm{~B}$ ) were therefore used for a series of induced fit simulations and molecular dockings-the final step of the workflow (Figure 1). 

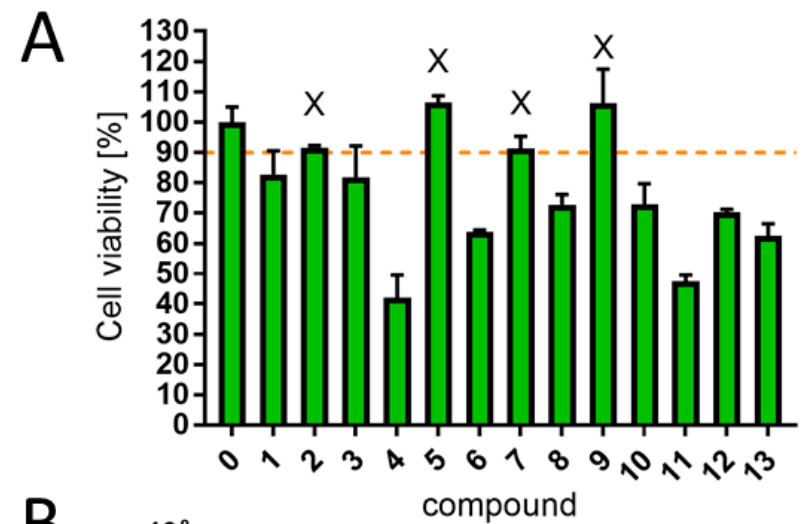

B

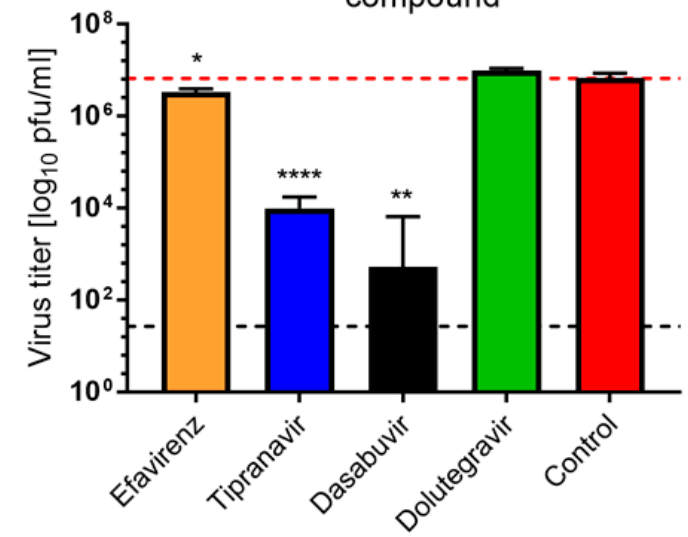

0 Control (non-infected cells)

1 Paritaprevir

2 Dolutegravir

3 Raltegravir potassium

4 Delavirdine mesylate

5 Efavirenz

6 Elvitegravir

7 Tipranavir

8 Saquinavir mesylate

9 Dasabuvir

10 Maraviroc

11 Trifluridine

12 Tauroursodeoxycholate sodium

13 Virus control

Figure 2. During the initial screening, all compounds identified by the molecular docking were tested at a single concentration of $50 \mu \mathrm{M}$ for inhibition of ZIKV-mediated cytopathic effect (CPE) (simultaneous treatment assay). Dimethyl sulfoxide (DMSO) was added to virus-infected cells as a negative control and to control, non-infected cells, at a concentration corresponding to a dilution of the initial drug-DMSO stock. Culture medium containing appropriate drug concentrations and virus inoculum (multiplicity of infection $=0.1$ ) was added to the cell monolayers. After $48 \mathrm{~h}$ of incubation at $37^{\circ} \mathrm{C}$, culture media were collected, and CPE was quantified using the Cell Counting Kit- 8 and expressed as percentage of cell viability. The horizontal dashed line indicates $90 \%$ cell viability compared to uninfected cells (A). Inhibitory effect of the compounds that reduced ZIKV-mediated CPE ( $>90 \%$ viability of the cells in the culture compared to the uninfected cells; marked by $\mathrm{X}$ in (A)) on ZIKV growth, as determined by plaque assay. Horizontal dashed line indicates the minimum detectable threshold of $1.44 \log _{10} \mathrm{pfu} / \mathrm{mL}$ (black), and the mean virus titer in control (untreated) cells (red). Data were analyzed by Student's $t$-test (GraphPad Prism, version 7.04); ${ }^{*}, p<0.05 ;{ }^{* *}, p<0.01 ;{ }^{* * * *}, p<0.0001$ (B).

None of the PDB non-nucleoside co-crystalized with flavivirus polymerases (Supplementary Table S1) indicate that efavirenz will bind at the HIV polymerase site (Figure 3A). Molecular docking and induced fit simulations also showed that efavirenz binding at the HIV position caused large, unnatural conformational changes at the palm domain (data not shown). We therefore focused on the average polymerase binding site (Figure $3 \mathrm{~A}$ ) for efavirenz simulations. Although both efavirenz and tipranavir approach their respective binding coordinates, the enthalpy values were slightly less favorable than their co-crystalized analogs (Figure 3C). This may be due to the structural-sequence diversity between HIV and flavivirus enzymes, indicating a distinct allosteric binding site for efavirenz and tipranavir, or that these antivirals may not be as effective for ZIKV (compared with HIV). 


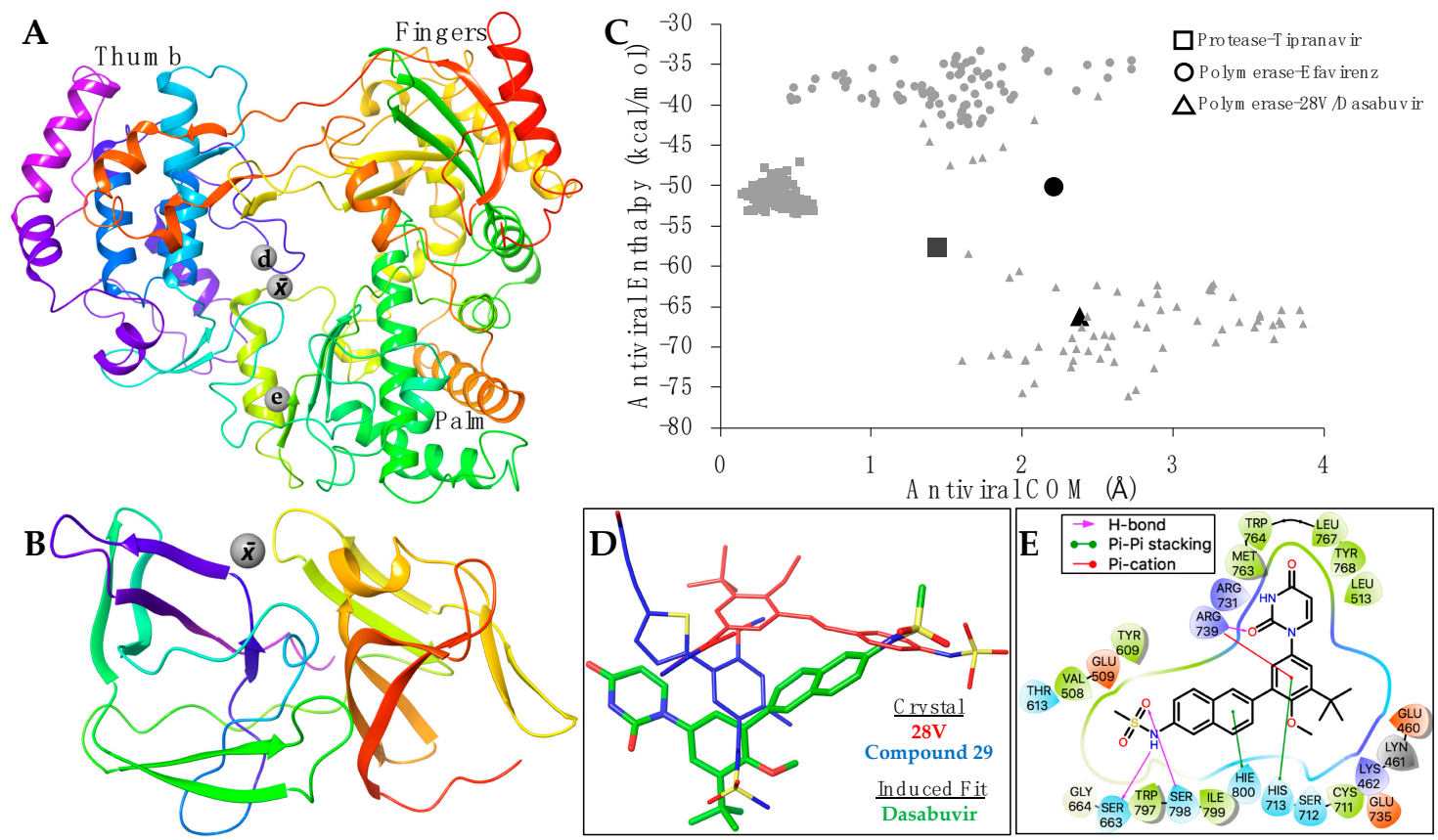

Figure 3. The PDB ZIKV polymerase (A; PDB: 5U0B) and protease (B; PDB: 5YOF) are color-coded from the amine-terminus (red) to the carboxyl-terminus (purple). The total average $(\bar{x})$, Dengue average (d), and HIV polymerase-efavirenz (e) binding coordinates are labeled and shown as grey spheres. The domains of ZIKV polymerase (A) are also labeled. The protein motion simulation results (C) show the enthalpy (y-axis) and the center of mass (COM) of the antivirals migration within its respective coordinates (x-axis). The darker/larger geometric shapes indicate average results for crystal structures of HIV protease-tipranavir (square; PDB: 6DIF), HIV polymerase-efavirenz (circle; PDB: 1FK9), and HCV polymerase-28V (triangle; PDB: 4MKB). The legend indicates the antiviral and target. The superposition (D) of co-crystalized antiviral structures 28V (red; PDB: 4MKB), compound 29 (blue; PDB: 5HMZ), and the best pose/iteration of ZIKV polymerase-dasabuvir (green). A ligand diagram (E) indicating the interacting ZIKV polymerase residues with dasabuvir and type of interaction (legend).

Of the three antivirals, however, dasabuvir clusters within the average enthalpy and coordinates of the analog $28 \mathrm{~V}$ co-crystalized with $\mathrm{HCV}$ polymerase (Figure $3 \mathrm{C}$ ). Although dasabuvir approximates the position of $28 \mathrm{~V}$, the coordination of dasabuvir resembles that of the non-nucleosides bound to Dengue polymerases (Figure 3A). The Dengue polymerase bound to compound 29 (PDB: 5HMZ) was used as an example (Figure 3D). Aside from the electrostatic interactions between ZIKV polymerase and dasabuvir, there are five residues that form direct contact (Figure 3E). Hydrogen bonds are formed at the methanesulfonamide group of dasabuvir with ZIKV polymerase residues Ser663 and Ser798, and Arg739 forms bonds with the dioxopyrimidine group. Pi-cation interactions are also coordinated by Arg739 along with the pi-pi stacking of His713 at the methoxyphenyl group. Lastly, His800 forms pi-pi stacking with naphthalene group of dasabuvir (Figure 3E).

We then evaluated the cytotoxic profiles and antiviral potency of efavirenz, tipranavir, and dasabuvir in detail using three cell lines: Vero cells, human neuroblastoma cells UKF-NB-4, and primary HBCAs. Both UKF-NB-4 and HBCA are target cells for neurotropic and neuroinvasive VBFs and therefore represent a clinically relevant model for our cytotoxicity/antiviral studies. In Vero cells, we observed no cytotoxicity across a concentration range $0-100 \mu \mathrm{M}$ in the case of tipranavir $\left(\mathrm{CC}_{50}>100 \mu \mathrm{M}\right.$; Table 2$)$. Dasabuvir and efavirenz exerted only moderate cytotoxicity in Vero cells ( $\mathrm{CC}_{50}$ values of 101.50 and $73.57 \mu \mathrm{M}$, respectively). For UKF-NB-4 cells, the highest toxicity was associated with dasabuvir $\left(\mathrm{CC}_{50}, 21.28 \mu \mathrm{M}\right)$, followed by efavirenz $\left(\mathrm{CC}_{50}, 31.85 \mu \mathrm{M}\right)$ and tipranavir $\left(\mathrm{CC}_{50}, 89.17 \mu \mathrm{M}\right)$. Of interest, the three drugs showed the highest cytotoxicity in HBCAs, with $\mathrm{CC}_{50}$ values of $25.98 \mu \mathrm{M}$ for dasabuvir, $34.03 \mu \mathrm{M}$ for tipranavir, and 16.68 $\mu \mathrm{M}$ for efavirenz (Table 3). 
We previously had observed a similar trend of selective toxicity (increasing toxicity as follows: Vero $<$ UKF-NB-4 < HBCA) in our study focused on the toxicity comparison of antiviral drug arbidol in multiple cell types [31]. Our hypothesis is that the selective toxicity can be a result of different levels of drug uptake by distinct cell types and/or different levels of enzymatic conversion of the compounds into toxic metabolites.

Table 2. Virus inhibition and cytotoxicity characteristics of efavirenz, tipranavir, and dasabuvir in Vero cells.

\begin{tabular}{|c|c|c|c|c|c|c|}
\hline & Virus (Strain) & $\begin{array}{c}\mathrm{EC}_{50} \\
(\mu \mathrm{M})^{\mathrm{a}}\end{array}$ & $95 \% \mathrm{CI}(\mu \mathrm{M})^{\mathrm{b}}$ & $\begin{array}{l}\mathrm{CC}_{50} \\
(\mu \mathrm{M})^{\mathrm{c}}\end{array}$ & $95 \% C I(\mu M)^{b}$ & $\begin{array}{c}\text { Selectivity } \\
\text { Index (SI) } \\
\left(\mathrm{CC}_{50} / \mathrm{EC}_{50}\right)\end{array}$ \\
\hline \multirow{5}{*}{ Efavirenz } & ZIKV (Paraiba_01) & 25.78 & $23.54-28.03$ & \multirow{5}{*}{73.57} & \multirow{5}{*}{$64.27-87.43$} & 2.85 \\
\hline & ZIKV (MR-766) & 30.41 & $29.72-31.10$ & & & 2.41 \\
\hline & TBEV (Hypr) & 15.86 & $14.17-17.54$ & & & 4.63 \\
\hline & WNV (Eg101) & 21.94 & $19.58-24.30$ & & & 3.35 \\
\hline & WNV (13-104) & 16.32 & $14.79-17.86$ & & & 4.50 \\
\hline \multirow{5}{*}{ Tipranavir } & ZIKV (Paraiba_01) & 29.85 & $25.59-34.11$ & \multirow{5}{*}{134.90} & \multirow{5}{*}{$130.50-143.30$} & 4.51 \\
\hline & ZIKV (MR-766) & 30.29 & $27.68-32.90$ & & & 4.45 \\
\hline & TBEV (Hypr) & 35.54 & $32.98-38.10$ & & & 3.79 \\
\hline & WNV (Eg101) & 32.70 & $30.61-34.78$ & & & 4.12 \\
\hline & WNV (13-104) & 24.17 & $21.07-27.27$ & & & 5.58 \\
\hline \multirow{5}{*}{ Dasabuvir } & ZIKV (Paraiba_01) & 16.12 & $14.27-17.97$ & \multirow{5}{*}{101.50} & \multirow{5}{*}{$95.02-113.50$} & 6.29 \\
\hline & ZIKV (MR-766) & 15.50 & 11.00-19.99 & & & 6.54 \\
\hline & TBEV (Hypr) & 15.20 & $8.41-21.99$ & & & 6.67 \\
\hline & WNV (Eg101) & 18.12 & $17.06-19.18$ & & & 5.60 \\
\hline & WNV (13-104) & 15.65 & $12.68-18.62$ & & & 6.48 \\
\hline
\end{tabular}

${ }^{\mathrm{a}} \mathrm{EC}_{50}(50 \%$ effective concentration) values were calculated using GraphPad Prism (version 7.04, GraphPad Software, San Diego, CA, USA) as a nonlinear regression (agonist vs. normalized response) from three independent experiments done in triplicate. ${ }^{b} 95 \% \mathrm{CI} ; 95 \%$ confidence interval. ${ }^{c} \mathrm{CC}_{50}$ values, representing the concentration of compound that reduced cell viability by $50 \%$, were calculated using GraphPad Prism as a nonlinear regression (inhibitor vs. normalized response, variable slope).

Table 3. Cytotoxicity characteristics of efavirenz, tipranavir, and dasabuvir in UKF-NB-4 and HBCA cells.

\begin{tabular}{cccc}
\hline & Cell Type & CC $_{\mathbf{5 0}}(\mu \mathbf{M})^{\mathbf{a}}$ & 95\% CI $(\mu \mathbf{M})^{\mathbf{b}}$ \\
\hline \multirow{2}{*}{ Efavirenz } & UKF-NB-4 & 31.85 & $29.93-33.79$ \\
& HBCA & 16.68 & $12.70-21.68$ \\
\hline \multirow{2}{*}{ Dasabuvir } & UKF-NB-4 & 21.28 & $20.13-22.48$ \\
& HBCA & 25.98 & $22.98-29.87$ \\
\hline \multirow{2}{*}{ Tipranavir } & UKF-NB-4 & 89.17 & $86.90-91.46$ \\
& HBCA & 34.03 & $30.28-38.17$ \\
\hline
\end{tabular}

${ }^{a} C_{50}$ values, representing the concentration of compound that reduced cell viability by $50 \%$, were calculated using GraphPad Prism (version 7.04, GraphPad Software, San Diego, CA, USA) as a nonlinear regression (inhibitor vs. normalized response, variable slope). ${ }^{\text {b } 95 \% ~ C I ; ~} 95 \%$ confidence interval.

As the antiviral effect of the compounds was most pronounced using the pretreatment assays, we used this treatment regimen for all further antiviral analyses. We evaluated the antiviral effects of efavirenz, tipranavir, and dasabuvir in Vero cells against two representatives of mosquito-borne flaviviruses, ZIKV and WNV, and one representative of tick-borne flavivirus (TBEV) (pretreatment assay). All three inhibitors exhibited micromolar antiviral potency against all VBFs tested and reduced viral titers in a dose-dependent manner (Figure 4; Table 2). 
A

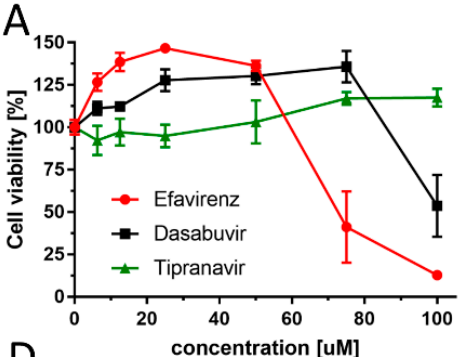

$\mathrm{D}$
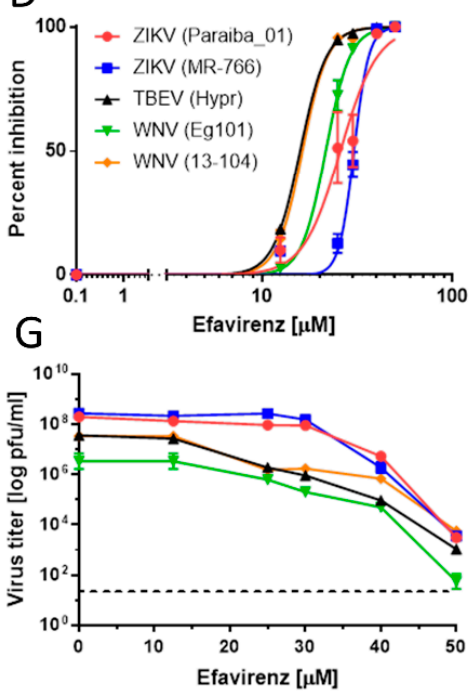

$B$
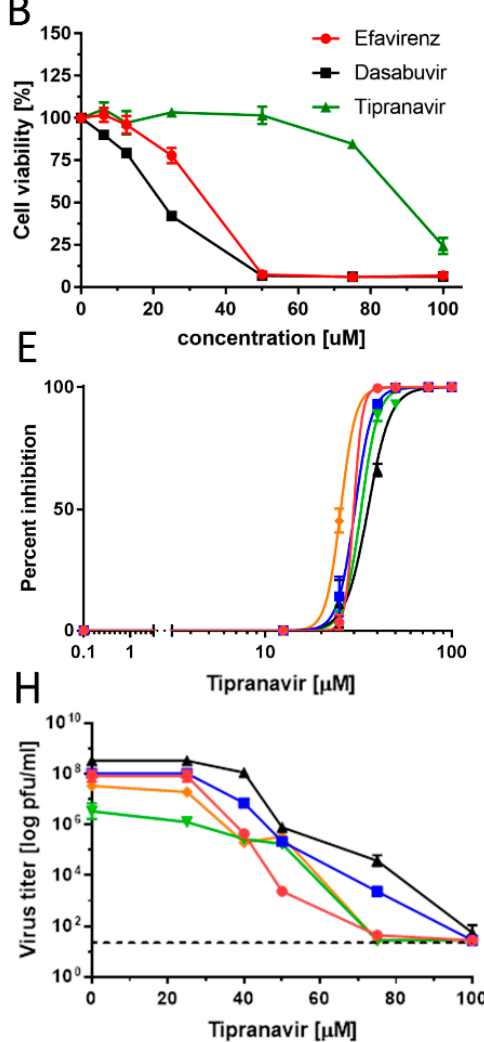

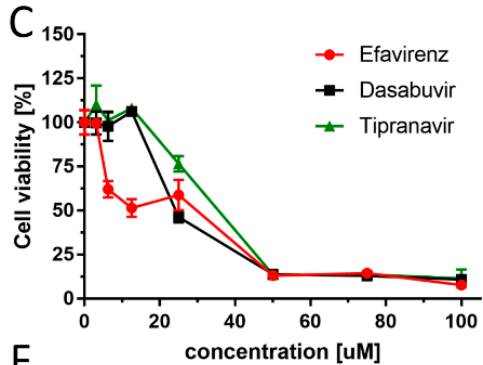

$\mathrm{F}$
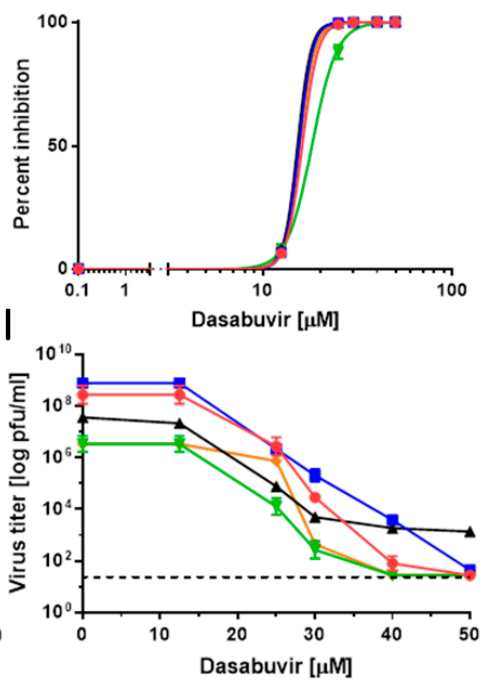

Figure 4. Cytotoxicity of efavirenz, tipranavir, and dasabuvir in Vero (A), UKF-NB-4 (B), and Human brain cortical astrocyte (HBCA) (C) cells, within the compound concentration range 0-100 $\mu \mathrm{M}$, at $72 \mathrm{~h}$ post-treatment. (D-F) Virus titer inhibition curves of the indicated flaviviruses in Vero cells in the presence of serial dilutions of efavirenz (D), tipranavir (E), and dasabuvir (F) in a pretreatment assay. Dose-dependent effect of efavirenz (G), tipranavir $(\mathbf{H})$, and dasabuvir $(\mathbf{I})$ on virus titers in Vero cells in a pretreatment assay (legends as in $\mathbf{D}$ ).

Efavirenz inhibited all investigated viruses with $\mathrm{EC}_{50}$ values ranging from 15.86 to $30.41 \mu \mathrm{M}$. The strongest inhibitory effect was seen for TBEV $\left(\mathrm{EC}_{50}, 15.86 \mu \mathrm{M}\right)$ and $\mathrm{WNV}\left(\mathrm{EC}_{50}, 16.32\right.$ and $21.94 \mu \mathrm{M}$ for 13-104 and Eg101, respectively). The inhibitory effect of efavirenz against ZIKV was less pronounced, with $\mathrm{EC}_{50}$ values of 25.78 and $30.41 \mu \mathrm{M}$ for Paraiba_01 and MR-766, respectively. Tipranavir inhibited all VBFs tested with similar $\mathrm{EC}_{50}$ values, ranging from 24.17 (WNV 13-104) to $35.54 \mu \mathrm{M}$ (TBEV). Dasabuvir showed the strongest and most robust antiviral effect in Vero cells $\left(\mathrm{EC}_{50}\right.$ values from 15.20 (TBEV) to $18.82 \mu \mathrm{M}$ (WNV Eg101)) (Figure 4F,I; Table 2). We further confirmed the anti-VBF activity of efavirenz, tipranavir, and dasabuvir in a cell-based flavivirus immunostaining assay, which showed a dose-dependent inhibition of surface $\mathrm{E}$ antigen expression by all VBFs tested in Vero cells (Figure 5). 


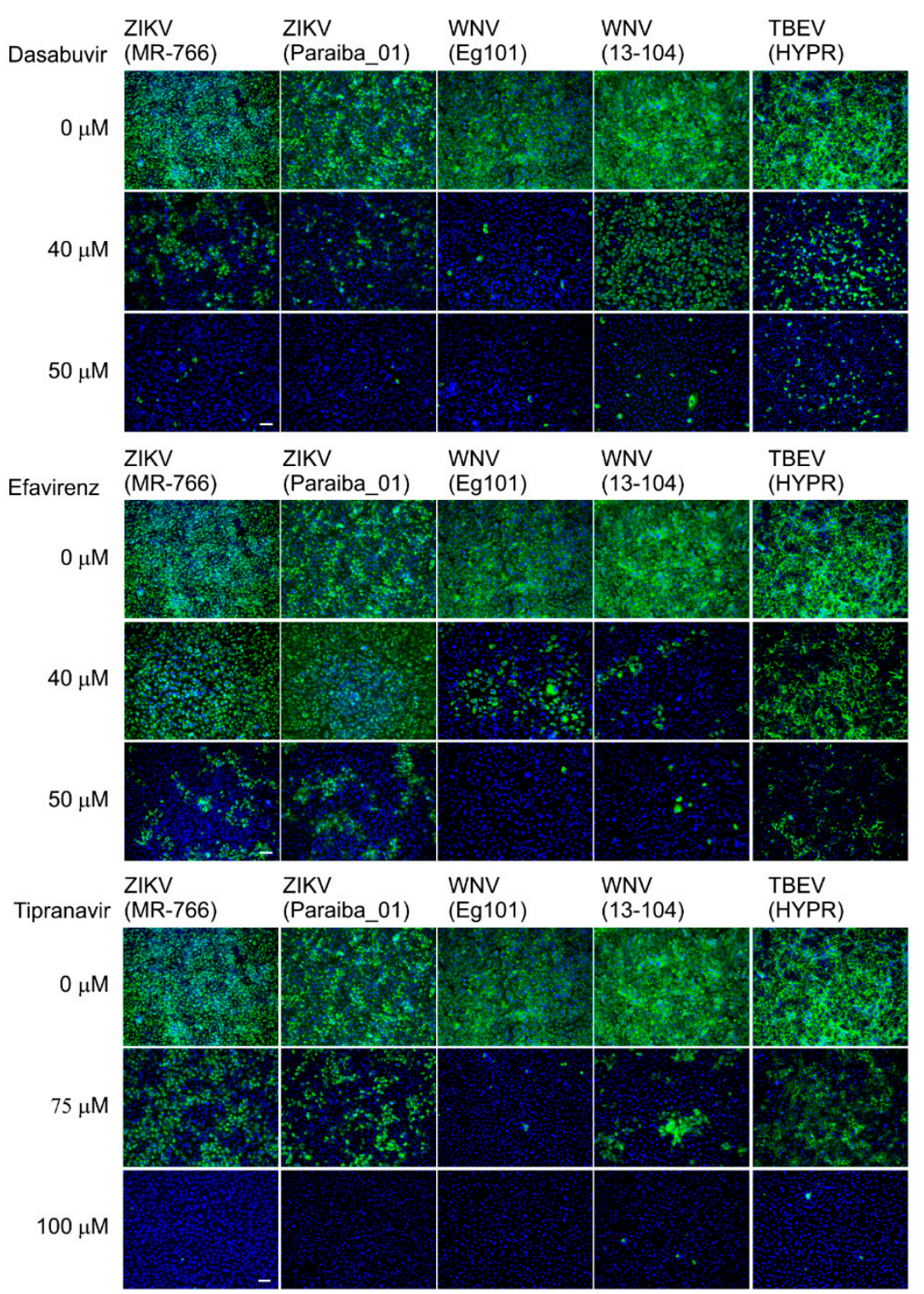

Figure 5. Inhibition of flaviviral surface E antigen expression by efavirenz, tipranavir, and dasabuvir. Virus-infected Vero cells were fixed on slides at $48 \mathrm{~h}$ p.i. and stained with flavivirus-specific antibody labeled with fluorescein isothiocyanate (FITC, green) and counterstained with 4',6-diamidino-2-phenylindole (DAPI, blue). Scale bar, $50 \mu \mathrm{m}$.

To investigate the antiviral effect of efavirenz, tipranavir, and dasabuvir in target cell types, primary HBCAs and human neuroblastoma cells (UKF-NB-4) were infected with Paraiba_01 strain of ZIKV and treated with serial dilutions of the drugs. At $48 \mathrm{~h}$ of incubation, we collected cell culture supernatants and quantified virus titer by plaque assay. In UKF-NB-4 cells, only tipranavir exhibited a significant antiviral effect against ZIKV (approx. $10^{3}$-fold at concentration $50 \mu \mathrm{M}$ ) (Figure 6C). The antiviral effect of dasabuvir was very low in these cells, resulting in a virus titer reduction of approximately $1 \log _{10} \mathrm{pfu} / \mathrm{mL}$ at the highest concentration of the drug in comparison with mock-treated control cells (Figure 6B). No antiviral effect of efavirenz was observed in UKF-NB-4 and HBCA cells at any concentration tested (Figure 6A,D). In ZIKV-infected HBCAs, treatment with dasabuvir significantly suppressed ZIKV replication in cell culture in a dose-dependent manner; treatment with $12.5 \mu \mathrm{M}$ of dasabuvir completely suppressed ZIKV replication in the cell culture (Figure 6E). Similarly, treatment with tipranavir significantly reduced ZIKV growth in HBCAs in a dose-dependent manner; treatment with $12.5 \mu \mathrm{M}$ of tipranavir reduced ZIKV replication in the HBCA culture by about $10^{3}$-fold (Figure $6 \mathrm{~F}$ ). 

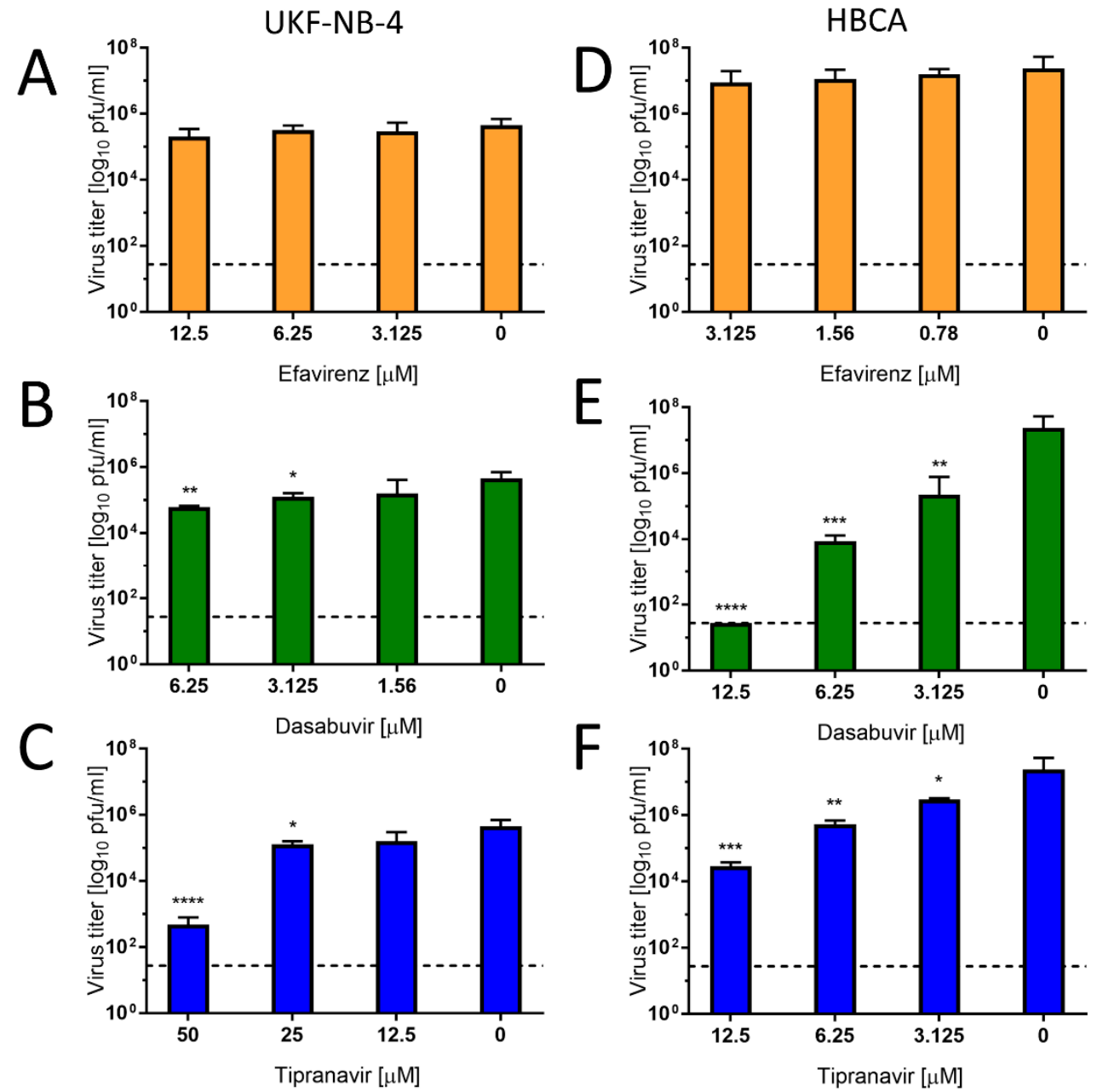

Figure 6. Inhibitory effect of efavirenz, tipranavir, and dasabuvir against ZIKV, strain Paraiba_01 in UKF-NB-4 (A,B, and C) and HBCA (C,D, and E) cells using a pretreatment assay. The cells were treated with serial dilutions of the drugs. The highest drug concentration represented the highest non-toxic concentrations for the particular cell type. Culture supernatants were collected at $48 \mathrm{~h}$ p.i., and the viral titers were determined by plaque assay. Horizontal dashed lines indicate the minimum detectable threshold of $1.44 \log _{10} \mathrm{pfu} / \mathrm{mL}$. Data were analyzed using Student's $t$-test (GraphPad Prism, version $7.04) ;^{*}, p<0.05 ;{ }^{* *}, p<0.01 ;{ }^{* * *}, p<0.001 ;{ }^{* * * *}, p<0.0001$.

Overall, the highest and most robust antiviral effect was observed for tipranavir in human neural cells. Dasabuvir exhibited anti-ZIKV effects in astrocytes and a slight effect in neuroblastoma cells, while efavirenz had no or negligible anti-ZIKV activity in either astrocytes or neuroblastoma cells. This result indicates a cell-type-dependent activity of dasabuvir and efavirenz, which may result from differences in drug uptake or metabolic processing by different cell types. We previously observed similar cell-type-dependent activities in case of anti-VBF activities of arbidol, when the antiviral effect was found to be substantial only in HBCA and Vero cells [31], as well as for several nucleoside analogues, whose antiviral effect was studied in PS and UKF-NB-4 cells [32].

Repurposing of approved drugs could accelerate the development of novel therapeutic strategies, particularly for emerging life-threatening infections for which therapies are lacking [33]. In such cases, broad-spectrum antiviral drugs with effectiveness against a wide range of viral species are extremely suitable. Previous studies aimed to discover potential anti-ZIKV therapeutics via a drug-repurposing screen. The results involved different hits from the screens, and no common compounds were identified among the studies $[6,7,34]$. In the current work, we identified three FDA-approved drugs-tipranavir, 
dasabuvir, and efavirenz-that exert antiviral activities against multiple flaviviruses in vitro and that were not identified in the previous anti-ZIKV screening studies.

A sulfonamide-containing dihydropyrone tipranavir is a non-peptidomimetic protease inhibitor currently used in combination with ritonavir to treat HIV-1 infections. It is advantageous particularly in treatment-experienced patients infected with protease inhibitor-resistant HIV-1 strains [35]. Tipranavir exhibits low-nanomolar anti-HIV activity in $\mathrm{H} 9$ cells $\left(\mathrm{EC}_{90}, 0.1 \mu \mathrm{M}\right)$, and for multidrug-resistant $\mathrm{HIV}$ isolates, the $\mathrm{EC}_{90}$ values range from 0.31 to $0.86 \mu \mathrm{M}[35,36]$. To the best of our knowledge, the current work is the first study that demonstrates its activity against flaviviruses.

Dasabuvir (previously known as ABT-333) is an inhibitor of the hepatitis $\mathrm{C}$ virus (HCV) and approved for use in combination with ombitasvir/paritaprevir/ritonavir for the treatment of chronic HCV infection $[37,38]$. In the HCV subgenomic replicon system, dasabuvir inhibits genotype 1a and $1 \mathrm{~b}$ replicons with $\mathrm{EC}_{50}$ values of 7.7 and $1.8 \mathrm{nM}$, respectively [30]. In our in vitro assays, dasabuvir had a micromolar $\mathrm{EC}_{50}$, which was comparable to other small molecule inhibitors that showed effectiveness in laboratory animals infected with VBFs $[25-27,39,40]$. The antiviral activity of dasabuvir was demonstrated regardless if applied before infection, at the time of infection, or post-infection (Figures 2B and 4, Supplementary Figure S1). The mechanism of action of dasabuvir is based on its interaction with HCV NS5B (an NS5B non-nucleoside polymerase inhibitor), leading to premature termination of synthesis of viral RNA genome [41]. Considering the structural similarities of HCV and VBF RNA-dependent RNA polymerases [42], the mechanism of action of dasabuvir could be analogous.

Efavirenz is a non-nucleoside inhibitor that also targets HIV reverse transcriptase. In combination with other antiretroviral drugs, this agent significantly reduces HIV viral load, attenuating or preventing damage to the immune system and reducing the risk of developing AIDS [34]. The 90-95\% inhibitory concentration of efavirenz for wild-type laboratory-adapted HIV strains and clinical isolates ranges from 1.7 to $25 \mathrm{nM}$ when cultivated in lymphoblastoid cell lines, macrophage/monocyte cultures, and peripheral blood mononuclear cells. Efavirenz demonstrates in vitro synergistic activity against HIV-1 in combination with zidovudine, indinavir, or didanosine [43].

In summary, our present study yielded three major findings. First, our results identified three FDA-approved drugs-efavirenz, tipranavir, and dasabuvir-that inhibit replication of multiple flaviviruses in vitro in Vero cells. All three inhibitors exhibited micromolar antiviral potency against all viruses tested and reduced viral titers in a dose-dependent manner. Secondly, the anti-ZIKV effect of these drugs in Vero cells was demonstrated regardless if the compounds were applied before infection, at the time of infection, or even post-infection. Third, the anti-ZIKV effect of dasabuvir and tipranavir was confirmed also in human neural cells, which represent target cell types for the virus. One of possible limitations of our study is the fact that the mechanism of action of the identified compounds remains elusive. Future studies are also needed to investigate the anti-flavivirus effect of the identified compounds in vivo.

\section{Conclusions}

Efavirenz, tipranavir, and dasabuvir at micromolar concentrations were identified to inhibit two representatives of mosquito-borne flaviviruses (ZIKV and WNV) and one representative of flaviviruses transmitted by ticks (TBEV). All foregoing results do not necessarily indicate that efavirenz, tipranavir, and dasabuvir are suitable candidates for treating humans infected by VBFs. These results, however, do identify novel activities. Further research on these FDA-approved antiviral drugs, either individually or in combination, will consider them as possible pan-flavivirus inhibitors.

Supplementary Materials: The following are available online at http://www.mdpi.com/2076-2607/8/4/599/s1, Supplementary Material S1: Configuration file and script containing information on the grid box centers and sizes of prepared receptors and selected drugs. Supplementary Material S2: The modifications made on the ligand refinement ready-made script for the induced fit simulations in PELE. Supplementary Table S1: PDB accession numbers indicated by target-location. Supplementary Figure S1: Inhibitory effect of efavirenz, tipranavir, and dasabuvir on ZIKV growth in Vero cells in a post-treatment assay. Supplementary Figure S2: Inhibitory effect of a reference compound (7-deaza-2'-C-methyladenosine) on ZIKV growth in Vero cells. 
Author Contributions: Conceptualization: M.S., J.J.V., F.C.E., L.E., and D.R.; methodology: M.S., J.J.V., F.C.E., J.H., I.C.U., M.F., J.S., L.E., and D.R.; supervision: F.C.E., J.J.V., L.E., and D.R.; draft preparation: M.S., J.J.V., F.C.E., L.E., and D.R.; manuscript revision: all authors. All authors have read and agreed to the published version of the manuscript.

Funding: The authors acknowledge financial support from the Ministry of Health of the Czech Republic (grant No. 16-34238A) and Ministry of Education, Youth and Sports of the Czech Republic (project "FIT" Pharmacology, Immunotherapy, nanoToxicology; grant No. CZ.02.1.01/0.0/0.0/15_003/0000495).

Conflicts of Interest: The authors declare no conflict of interest. The funders had no role in the design of the study; in the collection, analyses, or interpretation of data; in the writing of the manuscript, or in the decision to publish the results.

\section{References}

1. Simmonds, P.; Becher, P.; Bukh, J.; Gould, E.A.; Meyers, G.; Monath, T.; Muerhoff, S.; Pletnev, A.; Rico-Hesse, R.; Smith, D.B.; et al. Ictv Report Consortium. ICTV Virus Taxonomy Profile: Flaviviridae. J. Gen. Virol. 2017, 98, 2-3. [CrossRef] [PubMed]

2. Deval, J.; Symons, J.A.; Beigelman, L. Inhibition of viral RNA polymerases by nucleoside and nucleotide analogs: Therapeutic applications against positive-strand RNA viruses beyond hepatitis $C$ virus. Curr. Opin. Virol. 2014, 9, 1-7. [CrossRef] [PubMed]

3. Baier, A. Flaviviral infections and potential targets for antiviral therapy. In Flavivirus Encephalitis, 1st ed.; Ruzek, D., Ed.; InTech: Rijeka, Croatia, 2011; pp. 89-104.

4. Lazear, H.M.; Stringer, E.M.; de Silva, A.M. The Emerging Zika Virus Epidemic in the Americas Research Priorities. JAMA-J. Am. Med. Assoc. 2016, 315, 1945-1946. [CrossRef] [PubMed]

5. Han, Y.; Mesplède, T.; Xu, H.; Quan, Y.; Wainberg, M.A. The antimalarial drug amodiaquine possesses anti-ZIKA virus activities. J. Med. Virol. 2018, 90, 796-802. [CrossRef] [PubMed]

6. Adcock, R.S.; Chu, Y.K.; Golden, J.E.; Chung, D.H. Evaluation of anti-Zika virus activities of broad-spectrum antivirals and NIH clinical collection compounds using a cell-based, high-throughput screen assay. Antivir. Res. 2017, 138, 47-56. [CrossRef]

7. Barrows, N.J.; Campos, R.K.; Powell, S.T.; Prasanth, K.R.; Schott-Lerner, G.; Soto-Acosta, R.; Galarza-Muñoz, G.; McGrath, E.L.; Urrabaz-Garza, R.; Gao, J.; et al. A Screen of FDA-Approved Drugs for Inhibitors of Zika Virus Infection. Cell Host. Microbe. 2016, 20, 259-270. [CrossRef]

8. Madrid, P.B.; Chopra, S.; Manger, I.D.; Gilfillan, L.; Keepers, T.R.; Shurtleff, A.C.; Green, C.E.; Iyer, L.V.; Dilks, H.H.; Davey, R.A.; et al. A systematic screen of FDA-approved drugs for inhibitors of biological threat agents. PLoS ONE 2013, 8, e60579. [CrossRef]

9. Pascoalino, B.S.; Courtemanche, G.; Cordeiro, M.T.; Gil, L.H.; Freitas-Junior, L. Zika antiviral chemotherapy: Identification of drugs and promising starting points for drug discovery from an FDA-approved library. F1000Research 2016, 5, 2523. [CrossRef]

10. Berman, H.M.; Westbrook, J.; Feng, Z.; Gilliland, G.; Bhat, T.N.; Weissig, H.; Shindyalov, I.N.; Bourne, P.E. The Protein Data Bank. Nucleic Acids Res. 2000, 28, 235-242. [CrossRef]

11. Pettersen, E.F.; Goddard, T.D.; Huang, C.C.; Couch, G.S.; Greenblatt, D.M.; Meng, E.C.; Ferrin, T.E. UCSF Chimera-a visualization system for exploratory research and analysis. J. Comput. Chem. 2004, 25, 1605-1612. [CrossRef]

12. Sanner, M.F. Python: A programming language for software integration and development. J. Mol. Graph. Model. 1999, 17, 57-61. [PubMed]

13. Morris, G.M.; Huey, R.; Lindstrom, W.; Sanner, M.F.; Belew, R.K.; Goodsell, D.S.; Olson, A.J. AutoDock4 and AutoDockTools4: Automated docking with selective receptor flexibility. J. Comput. Chem. 2009, 30, 2785-2791. [CrossRef] [PubMed]

14. Wishart, D.S.; Knox, C.; Guo, A.C.; Cheng, D.; Shrivastava, S.; Tzur, D.; Gautam, B.; Hassanali, M. DrugBank: A knowledgebase for drugs, drug actions and drug targets. Nucleic Acids Res. 2008, 36, D901-D906. [CrossRef] [PubMed]

15. O’Boyle, N.M.; Banck, M.; James, C.A.; Morley, C.; Vandermeersch, T.; Hutchison, G.R. Open Babel: An open chemical toolbox. J. Cheminform. 2011, 3, 33. [CrossRef] [PubMed]

16. Irwin, J.J.; Sterling, T.; Mysinger, M.M.; Bolstad, E.S.; Coleman, R.G. ZINC: A free tool to discover chemistry for biology. J. Chem. Inf. Model. 2012, 52, 1757-1768. [CrossRef] [PubMed] 
17. Trott, O.; Olson, A.J. AutoDock Vina: Improving the speed and accuracy of docking with a new scoring function, efficient optimization, and multithreading. J. Comput. Chem. 2010, 31, 455-461. [CrossRef]

18. Keshavarz, F.; Alavianmehr, M.M.; Yousefi, R. Molecular interaction of Benzalkonium Ibuprofenate and its discrete ingredients with human serum albumin. Phys. Chem. Res. 2013, 1, 111-116.

19. Sastry, G.M.; Adzhigirey, M.; Day, T.; Annabhimoju, R.; Sherman, W. Protein and ligand preparation: Parameters, protocols, and influence on virtual screening enrichments. J. Comput.-Aided Mol. Des. 2013, 27, 221-234. [CrossRef]

20. Madadkar-Sobhani, A.; Guallar, V. PELE web server: Atomistic study of biomolecular systems at your fingertips. Nucleic Acids Res. 2013, 41, W322-W328. [CrossRef]

21. Borrelli, K.W.; Vitalis, A.; Alcantara, R.; Guallar, V. PELE: Protein Energy Landscape Exploration. A Novel Monte Carlo Based Technique. J. Chem. Theory Comput. 2005, 1, 1304-1311. [CrossRef]

22. Jorgensen, W.L.; Maxwell, D.S.; Tirado-Rives, J. Development and Testing of the OPLS All-Atom Force Field on Conformational Energetics and Properties of Organic Liquids. J. Am. Chem. Soc. 1996, 118, 11225-11236. [CrossRef]

23. De Madrid, A.T.; Porterfield, J.S. A simple micro-culture method for the study of group B arboviruses. Bull. World Health Organ. 1969, 40, 113-121.

24. Eyer, L.; Valdés, J.J.; Gil, V.A.; Nencka, R.; Hřebabecký, H.; Šála, M.; Salát, J.; Černý, J.; Palus, M.; De Clercq, E.; et al. Nucleoside inhibitors of tick-borne encephalitis virus. Antimicrob. Agents Chemother. 2015, 59, 5483-5493. [CrossRef] [PubMed]

25. Eyer, L.; Fojtíková, M.; Nencka, R.; Rudolf, I.; Hubálek, Z.; Ruzek, D. Viral RNA-Dependent RNA Polymerase Inhibitor 7-Deaza-2'-C-Methyladenosine Prevents Death in a Mouse Model of West Nile Virus Infection. Antimicrob. Agents Chemother. 2019, 63, e02093-18. [CrossRef]

26. Eyer, L.; Nencka, R.; Huvarová, I.; Palus, M.; Joao Alves, M.; Gould, E.A.; De Clercq, E.; Růžek, D. Nucleoside Inhibitors of Zika Virus. J. Infect. Dis. 2016, 214, 707-711. [CrossRef] [PubMed]

27. Zmurko, J.; Marques, R.E.; Schols, D.; Verbeken, E.; Kaptein, S.J.; Neyts, J. The Viral Polymerase Inhibitor 7-Deaza-2'-C-Methyladenosine Is a Potent Inhibitor of In Vitro Zika Virus Replication and Delays Disease Progression in a Robust Mouse Infection Model. PLoS Negl. Trop. Dis. 2016, 10, e0004695. [CrossRef] [PubMed]

28. Ren, J.; Milton, J.; Weaver, K.L.; Short, S.A.; Stuart, D.I.; Stammers, D.K. Structural basis for the resilience of efavirenz (DMP-266) to drug resistance mutations in HIV-1 reverse transcriptase. Structure 2000, 8, 1089-1094. [CrossRef]

29. Wong-Sam, A.; Wang, Y.F.; Zhang, Y.; Ghosh, A.K.; Harrison, R.W.; Weber, I.T. Drug Resistance Mutation L76V Alters Nonpolar Interactions at the Flap-Core Interface of HIV-1 Protease. ACS Omega 2018, 3, 12132-12140. [CrossRef]

30. Kati, W.; Koev, G.; Irvin, M.; Beyer, J.; Liu, Y.; Krishnan, P.; Reisch, T.; Mondal, R.; Wagner, R.; Molla, A.; et al. In vitro activity and resistance profile of dasabuvir, a nonnucleoside hepatitis $\mathrm{C}$ virus polymerase inhibitor. Antimicrob. Agents Chemother. 2015, 59, 1505-1511. [CrossRef]

31. Haviernik, J.; Štefánik, M.; Fojtíková, M.; Kali, S.; Tordo, N.; Rudolf, I.; Hubálek, Z.; Eyer, L.; Ruzek, D. Arbidol (Umifenovir): A Broad-Spectrum Antiviral Drug That Inhibits Medically Important Arthropod-Borne Flaviviruses. Viruses 2018, 10, E184. [CrossRef]

32. Eyer, L.; Smidkova, M.; Nencka, R.; Neča, J.; Kastl, T.; Palus, M.; De Clercq, E.; Růžek, D. Structure-activity relationships of nucleoside analogues for inhibition of tick-borne encephalitis virus. Antivir. Res. 2016, 133, 119-129. [CrossRef] [PubMed]

33. Wang, S.; Liu, Y.; Guo, J.; Wang, P.; Zhang, L.; Xiao, G.; Wang, W. Screening of FDA-Approved Drugs for Inhibitors of Japanese Encephalitis Virus Infection. J. Virol. 2017, 91, e01055-17. [CrossRef]

34. Xu, M.; Lee, E.M.; Wen, Z.; Cheng, Y.; Huang, W.K.; Qian, X.; Tcw, J.; Kouznetsova, J.; Ogden, S.C.; Hammack, C.; et al. Identification of small-molecule inhibitors of Zika virus infection and induced neural cell death via a drug repurposing screen. Nat. Med. 2016, 22, 1101-1107. [CrossRef] [PubMed]

35. Schapiro, J.M.; Scherer, J.; Boucher, C.A.; Baxter, J.D.; Tilke, C.; Perno, C.F.; Maggiolo, F.; Santoro, M.M.; Hall, D.B. Improving the prediction of virological response to tipranavir: The development and validation of a tipranavir-weighted mutation score. Antivir. Ther. 2010, 15, 1011-1019. [CrossRef] [PubMed]

36. Luna, B.; Townsend, M.U. Tipranavir: The first nonpeptidic protease inhibitor for the treatment of protease resistance. Clin Ther. 2007, 29, 2309-2318. [CrossRef] [PubMed] 
37. King, J.R.; Zha, J.; Khatri, A.; Dutta, S.; Menon, R.M. Clinical Pharmacokinetics of Dasabuvir. Clin. Pharmacokinet. 2017, 56, 1115-1124. [CrossRef]

38. Trivella, J.P.; Gutierrez, J.; Martin, P. Dasabuvir: A new direct antiviral agent for the treatment of hepatitis C. Expert Opin Pharmacother. 2015, 16, 617-624. [CrossRef]

39. Julander, J.G.; Bantia, S.; Taubenheim, B.R.; Minning, D.M.; Kotian, P.; Morrey, J.D.; Smee, D.F.; Sheridan, W.P.; Babu, Y.S. BCX4430, a novel nucleoside analog, effectively treats yellow fever in a Hamster model. Antimicrob Agents Chemother. 2014, 58, 6607-6614. [CrossRef]

40. Eyer, L.; Kondo, H.; Zouharova, D.; Hirano, M.; Valdés, J.J.; Muto, M.; Kastl, T.; Kobayashi, S.; Haviernik, J.; Igarashi, M.; et al. Escape of Tick-Borne Flavivirus from 2'-C-Methylated Nucleoside Antivirals Is Mediated by a Single Conservative Mutation in NS5 That Has a Dramatic Effect on Viral Fitness. J. Virol. 2017, 91, e01028-17. [CrossRef]

41. Gentile, I.; Buonomo, A.R.; Borgia, G. Dasabuvir: A Non-Nucleoside Inhibitor of NS5B for the Treatment of Hepatitis C Virus Infection. Rev. Recent Clin. Trials. 2014, 9, 115-123. [CrossRef]

42. Černý, J.; Černá Bolfíková, B.; Valdés, J.J.; Grubhoffer, L.; Růžek, D. Evolution of tertiary structure of viral RNA dependent polymerases. PLoS ONE 2014, 9, e96070. [CrossRef] [PubMed]

43. Duarte, H.; Cruz, J.P.; Aniceto, N.; Ribeiro, A.C.; Fernandes, A.; Paixão, P.; Antunes, F.; Morais, J. Population Approach to Efavirenz Therapy. J. Pharm. Sci. 2017, 106, 3161-3166. [CrossRef] [PubMed]

(C) 2020 by the authors. Licensee MDPI, Basel, Switzerland. This article is an open access article distributed under the terms and conditions of the Creative Commons Attribution (CC BY) license (http://creativecommons.org/licenses/by/4.0/). 